\title{
Realidad virtual y simulación en el entrenamiento de los estudiantes de medicina
}

\author{
G. Vázquez-Mata
}

\section{Necesidad de un cambio en las metodologías educativas aplicadas en medicina}

La práctica de la medicina tiene actualmente algunos aspectos diferenciadores en relación a etapas anteriores. Entre estos aspectos cabe destacar: el trabajo en equipo multidisciplinario, la simultaneidad de múltiples datos de distintas fuentes, la toma de decisiones complejas y con niveles de incertidumbre, competencias diferentes que deben de converger en una misma acción, y la participación activa del paciente/ciudadano en la toma de decisiones que le atañen.

Merecen mención aparte la incorporación de las tecnologías de la información y de la comunicación (TIC), los cambios culturales y una nueva realidad social, el incremento progresivo de la población de enfermos crónicos crecientes y de población frágil por su edad, que suponen un enfoque distinto del de los enfermos agudos.

La suma de estas facetas de la medicina obliga a replantear el perfil del medico, del enfermero o de cualquier otro profesional de la salud. Este nuevo profesional deberá reciclarse permanentemente o incluso poder cambiar de área de actividad; tiene que saber enfrentarse a nuevos problemas y dar respuestas objetivas y ajustadas a las necesidades del paciente. Sus competencias van allá de las meramente relacionas con las enfermedades; así, requiere un buen dominio de la comunicación en todas sus vertientes, un profundo sentido del profesionalismo y un manejo de las TIC.
Para alcanza este perfil, el alumno, desde el primer momento, debe de ser el propio protagonista de su formación, relegando la función del profesor a una actividad tutorial y de mentor. Esta formación ha de asegurar que, cuando entre en contacto con el paciente, haya adquirido las habilidades necesarias que eviten a éste riesgos y molestias innecesarias. A su vez, los métodos de formación que se ofrezcan a los estudiantes deben de tener curvas de aprendizaje que mejoren su rendimiento.

Desde esta perspectiva, la clase magistral en su concepción actual -es decir, la clase basada en el profesor con alumnos como receptores pasivos y con bajo nivel de interactividad-pierde parte de su valor en la formación de los futuros profesionales. Nuevos espacios de formación con funciones específicas, nuevas tecnologías y nuevas metodologías constituyen el eje de este cambio de paradigma educativo que necesita la medicina en el s. XxI.

Todas las actividades educativas tienen actualmente un ciclo, con cuatro etapas: la primera es la acción educativa propiamente dicha; la segunda es el feedback (retroalimentación), en el que se analiza lo realizado y se compara con los objetivos que se habían predeterminado; la tercera etapa, denominada debriefing, corresponde al análisis critico y razonado de la acción educativoa para construir espacios de conocimiento nuevo; y, finalmente, existe la evaluación, que puede adquirir múltiples formas. Estas actividades han de realizarse bien contextualizadas, es decir, en condiciones que favorezcan su asimilación y formando parte de un currículo preestablecido.
Director de I+D+i. Centro de Simulación Médica Avanzada e Innovación Tecnológica. Parque Tecnológico de Ciencias de la Salud. Fundación IAVANTE. Junta de Andalucía.

E-mail guillermo.vazquez@ iavante.es 


\section{Simulación de la realidad como herramienta de cambio}

La tecnología moderna permite conjugar equipos de robótica, realidad virtual o simplemente recurriendo a actores, y escenarios que remeden la realidad, consiguiendo reproducir situaciones de manera muy real.

\section{Habilidades básicas}

Este concepto abarca habilidades individuales simples, que pueden ser tanto especificas como transversales, y que posteriormente tendrán con combinarse con habilidades complejas y actividades de equipo.

El entrenamiento se inicia con simulación de habilidades en maniquíes elementales, que corresponden a órganos o partes completas (p. ej., tórax) del cuerpo humano. Estos equipos permiten, por un lado, reconocer la estructura de dicho órgano, y por otra, la más importante, practicar determinadas habilidades manuales (p. ej., intubación, punciones, vías venosas, drenajes...). Equiparable a esta simulación de habilidades simples motrices es el aprendizaje del manejo de determinados equipos, como el desfibrilador, el respirador de transporte o un espirómetro.

Paralelamente, y al mismo nivel, están las habilidades comunicativas, que se ensayan con actores para la recogida de historias clínicas e información normal a las familias y al enfermo.

\section{Habilidades que requieren interactividad}

El eslabón inicial de estas simulaciones viene dado por maniquíes que, además de la reproducción anatómica, están dotados de un software que reproduce una función de algún órgano y que permite al estudiante aplicar sus conocimientos; el ejemplo más patente son los equipos en los cuales un tórax reproduce la semiología cardíaca y pulmonar con un máximo realismo. El estudiante puede identificar y discriminar entre distintas opciones e identificar el síndrome al que van ligados.

La reanimación cardiovascular, con las habilidades que cada una de sus fases requiere, así como su aplicación final conjunta por un equipo de alumnos, también puede considerarse como integrable en esta fase.

El hecho de escribir un informe/historia médico correctamente y de iniciar las hojas de pres- cripciones y órdenes medicas pueden englobarse en la fase inicial de simulación interactiva.

El segundo eslabón viene dado por la robótica interactiva compleja, en la que se reproduce un cuerpo humano completo, con un software que dota al muñeco de todas las funciones cardíacas, vasculares y pulmonares. Esto permite diseñar síndromes/casos clínicos completos: el estudiante debe explorar al robot, llegar a una orientación clínica e iniciar un conjunto de habilidades básicas si la situación lo requiere. A partir de aquí, el nivel de complejidad puede elevarse. La otra virtud de este tipo de entrenamiento es que puede realizarse en equipo, de manera que el estudiante aprenda las normas básicas que rigen la actividad en equipo.

Resulta importante iniciar el entrenamiento en situaciones de comunicación difícil, como pueden ser las malas noticias, las peticiones de consentimiento informado y la relación con pacientes de trato complejo.

También las actividades de role-play, simulando reuniones de trabajo, análisis de incidentes, etc., son útiles para que el estudiante conozca las técnicas de las reuniones en grupo y su importancia como soporte a la práctica clínica de calidad.

La realidad virtual ofrece diversas posibilidades en el entrenamiento médico. Esta tecnología se emplea para el entrenamiento de habilidades complejas como endoscopia, laparoscopia o navegación endovascular; en general, estas habilidades se dirigen más a la formación especializada que a la formación de grado.

\section{E-learning}

\section{Condiciones previas}

Las TIC deben formar parte del entrenamiento básico y avanzado de los estudiantes de medicina y de los profesionales en activo. Esta formación ha de abarcar dos vertientes: una, correspondiente a las diversas terminales propias de los usuarios y su manejo experto, y en segundo lugar, el manejo de las herramientas de interrelación entre usuarios, así como el de las bibliotecas digitales y la búsqueda de información en general. en todas sus facetas. Estas premisas contituyen la base para que las TIC puedan dar todo 
su valor añadido en el día a día de estudiantes y profesores.

\section{Las TIC en la enseñanza de grado}

\section{Clases magistrales}

La clase magistral en versión clásica se diseña basándose en el profesor y con un alumno que recibe información pasivamente. Este perfil arranca del siglo $\mathrm{xx}$, cuando el conocimiento estaba ligado principalmente a la experiencia del profesor; sin embargo, en la actualidad, la accesibilidad a la información de las bibliotecas presenciales o virtuales - por tanto, sin limites horarios ni geográficosaconseja redefinir la función de la clase. Las TIC permiten este cambio. Su aportación más notable puede ser la videoconferencia, que permite acercar a los mejores expertos para que aporten su opinión y experiencia en temas de máximo interés. El hecho de aproximar los expertos a los alumnos, además de transmitir su experiencia, cumple una función de modelaje, es decir, de copia de un estilo y una cultura de hacer la medicina. Estos expertos, especialmente cuando son de otros países, permiten visualizar la importancia de los idiomas en la cultura médica actual. Las herramientas de comunicación en tiempo real o diferido y las herramientas de trabajo colaborativo virtuales ofrecen un nivel de interactividad profesor/alumno que el aula presencial clásica nunca ha alcanzado.

\section{Talleres}

Esta herramienta permite el trabajo en grupos pequeños, normalmente no superiores a 12 personas, y dentro del cual el papel de profesor se transforma en el de tutor. La función del trabajo en talleres consiste en desarrollar el pensamiento crítico y reflexivo. En este ambiente, las pizarras interactivas (blackboards) y la consulta de las bibliotecas virtuales, tanto para buscar evidencia como para obtener respuestas a los temas tratados, se basan en las TIC. El trabajo colectivo mediante herramientas de comunicación y colaborativas se hacen imprescindibles. Las TIC tienen otra vez un protagonismo central.

También conviene que la telemedicina, la teleasistencia, la historia clínica informatizada y la receta electrónica se conozcan desde los últimos años de la carrera, de manera que se facilite su utilización al llegar a los hospitales..

\section{Bibliografía}

1. Fundación Iavante. Innovación docente vía TIC. Educ Med 2006; 9 (Supl): S3-64.

2. Fundación Iavante. URL: http://www.iavantefundacion. com/portal3d/default.htm. 